\title{
LA LOCALIZACIÓN SECTORIAL DEL COOPERATIVISMO: UNA APROXIMACIÓN A NIVEL TERRITORIAL ESPAÑOL
}

\author{
POR \\ Ma del Carmen PÉREZ GONZÁLEZ y \\ Lidia VALIENTE PALMA ${ }^{1}$
}

\section{RESUMEN}

La posibilidad de cambiar los modelos de desarrollo socioeconómicos hacia otros más sostenibles y responsables está siendo planteada tanto por parte de investigadores como de diferentes instituciones, con una importancia que se ha visto incrementada a partir de la última crisis. En este escenario, la economía social se presenta como una oportunidad de cambio y de contribución a estos nuevos modelos, dado los principios y valores que la definen y sobre los que basa su funcionamiento; por ello, es relevante la aplicación de políticas y estrategias encaminadas a desarrollar este sector empresarial, con particularidades muy significativas. Sin embargo, es necesario considerar los recursos o especificidades y potencialidades de cada ámbito territorial. En esta línea, en el presente trabajo, se ha diseñado un indicador que permite identificar en qué sectores económicos y territorios destaca la economía social, -específicamente referido a las cooperativas por ser sus entidades más representativas en España-, y si su papel es diferente del resto del tejido empresarial, aspecto a considerar a la hora de promover sus organizaciones y elemento clave para determinar medidas y políticas socioeconómicas que contribuyan a la mejora de los sistemas productivos locales, con mayor garantía de resultados. Dicho indicador -peso del cooperativismo $\left(W_{i j}\right)$ obtenido a partir de los coeficientes de localización sectorial respecto a la variable empleo -de las sociedades cooperativas y de la economía en general-, se ha aplicado al año 2013, con el objeto de determinar la importancia o el peso sectorial del cooperativismo en relación a la economía general en las diferentes comunidades autónomas españolas. Entre los resultados obtenidos puede destacarse la importancia relativa del cooperativismo en determinados sectores y regiones que, a priori, no presentaban indicios sobre esta relevancia, lo que pone de

1 Universidad de Cádiz, departamento de Economía General. Direcciones de correo electrónico: maricarmen.perez@uca.es, valientepalma@gmail.com.

REVESCO No 123 - Primer Cuatrimestre 2017 - ISSN: 1885-8031 - www.ucm.es/info/revesco

http://dx.doi.org/10.5209/REVE.54916

Fecha de recepción: 18/04/2016

Fecha de aceptación: 02/09/2016 
manifiesto la necesidad de análisis profundos sobre el comportamiento del sector en los diferentes ámbitos territoriales.

Palabras clave: economía social, cooperativas, desarrollo territorial, coeficiente de localización sectorial.

Claves Econlit: A13, O10, P13.

\title{
SECTORIAL LOCATION OF THE COOPERATIVES: AN APPROACH AT THE TERRITORIAL LEVEL IN SPAIN
}

\begin{abstract}
The possibility to change the development models towards sustainability and responsibility is being considered by both the researchers and the different institutions, especially since the last crisis. In this scenario, the social economy provides an opportunity for change and contribution to these new models due to the principles and values on which it bases its working; the implementation of policies and strategies in order to develop this sector with specific characteristics is therefore important. Nevertheless, the resources or characteristics and potentialities in each territorial area must be taken into account. In this line, an indicator to identify in which economic sectors and territories the social economy stands out -in particular of cooperative societies since they are their most representative organizations in Spain-, and if its role is different from the rest of the firms, is performed. It is important to consider these aspect to promote the organizations of social economy and to implement socioeconomic measures and policies that contribute to the improvement of local production systems, and to ensure better results. This indicator -weight of cooperative societies $\left(W_{i j}\right)^{-}$, on the basis of the coefficients of sectoral locations in 2013 -of cooperative societies and general economy- measured in terms of employ, has been applied in order to determine the importance or the weight of these societies in each economic sector with regard to the general economy in the Autonomous Communities of Spain. The relative importance of the cooperatives -regarding to general economy- in certain sectors and regions, which initially did not seem to prove this, is among the main results. This reflects the need for deep analysis of the behaviour of the cooperatives in the different territorial frameworks.
\end{abstract}

Keywords: social economy, cooperatives, territorial development, coefficient of sectoral location. 


\section{INTRODUCCIÓN}

En los últimos años se ha producido un incremento de los estudios sobre el potencial de la economía social para la generación de empleo, la inclusión social de determinados colectivos, la respuesta a nuevas necesidades sociales o la dinamización socioeconómica de los territorios (Melián y Campos, 2010; Coraggio, 2011; Demoustier 2011; Martín y Lejarriaga, 2011; Jordán, Antuñano y Fuentes, 2013; Guridi y Pérez de Mendiguren, 2014, entre otros). Todos ellos coinciden en la necesidad de abordar tales cuestiones desde el planteamiento de nuevos modelos socioeconómicos sostenibles y responsables y hacia una mejor redistribución de la renta y la riqueza. Es sobre esta idea principal de sostenibilidad social y medioambiental que pivotan las recomendaciones y medidas -la integración del medio ambiente en la planificación y gestión de políticas, la evolución de las modalidades de consumo o el fomento de la educación y concienciación hacia el desarrollo sostenible ${ }^{2}$ - de numerosos organismos internacionales, -como la Organización de las Naciones Unidas (ONU)-, y nacionales, como es en el caso español la aprobación de la Ley de Economía Sostenible de $2011^{3}$.

Los nuevos modelos socioeconómicos deben ser capaces de contemplar estos desafíos desde el ámbito local. Las iniciativas y actuaciones deben comenzar en el nivel territorial donde surgen las principales relaciones socioeconómicas. Se tratan de retos globales que deben ser abordados mediantes actuaciones locales, es decir, a partir de estrategias que emanen de las propias comunidades o actores locales. Es ante este contexto que cobran importancia las actuaciones de las organizaciones de la economía social que, desde su surgimiento en el siglo XIX, han ido demostrando su capacidad de adaptación a las diferentes problemáticas y contextos territoriales, destacando sobre todo por sus fines perseguidos y sus actuaciones sobre la base de unos principios y valores para alcanzarlos.

Sin embargo, para lograr esto y, a su vez, conseguir un mayor desarrollo del sector de la economía social, es necesario llevar a cabo estrategias y políticas para el fomento y desarrollo de sus organizaciones. Estas políticas deben tener en cuenta las especificidades y recursos endógenos territoriales y vertebrarse dentro de las estrategias de desarrollo de los

\footnotetext{
2 Son algunas de las recomendaciones contempladas en el Programa 21 de la Organización de las Naciones Unidas (ONU). Más información en http://www.un.org/spanish/esa/sustdev/agenda21/index.htm (Consultado el $7 / 04 / 2016)$.

${ }^{3}$ Ley 2/2011, de 4 de marzo, de Economía Sostenible, publicada en el Boletín Oficial del Estado (BOE) núm. 55 de 05 de Marzo de 2011.
}

REVESCO No 123 - Primer Cuatrimestre 2017 - ISSN: 1885-8031 - www.ucm.es/info/revesco 
sistemas productivos locales. Esto requiere de un conocimiento exhaustivo del peso de la economía social en el territorio que lleva a la necesidad de realizar diagnósticos a niveles regionales o locales para identificar o determinar la situación en cada uno de ellos. Tales diagnósticos deberían ser la base para la aplicación de políticas específicas encaminadas a desarrollar este sector, sin perjuicio de la existencia de políticas a niveles territoriales superiores para complementarlas. En esta línea, en el presente trabajo, se realiza una aportación a la determinación de la importancia y de la contribución de las organizaciones más representativas y numerosas de la economía social en España, -las sociedades cooperativas-, en las diferentes comunidades autónomas españolas dentro de los sistemas productivos territoriales, a partir del empleo generado por estas organizaciones.

El objetivo principal de esta investigación consiste, por tanto, en determinar el peso sectorial del empleo en las cooperativas a nivel regional permitiendo, de esta manera, identificar los sectores de actividad -primario, industria, construcción y servicios- más destacables hacia los que se dirige el cooperativismo en cada comunidad autónoma, siendo esto un elemento clave para la toma de decisiones, el diseño y la aplicación de medidas y políticas estratégicas territoriales. Además, este trabajo permite abrir futuras líneas de investigación encaminadas a determinar qué factores o características hacen que el peso relativo del cooperativismo sea significativo o no en determinados sistemas productivos y territorios, así como establecer las estrategias más adecuadas para su consolidación como agente de dinamización territorial. Las cuestiones de investigación a responder a partir de este objetivo principal son las siguientes: ¿se comporta el empleo generado por las sociedades cooperativas de la misma forma que el empleo en la economía general?, es decir, ¿se dirige hacia los mismos sectores económicos?; y si es así, ¿en qué medida? ¿Existe un único modelo de cooperativismo en España? Para ello se ha seguido la estructura que se expone a continuación.

En primer lugar, se establece un marco teórico donde se destaca la importancia y la relación entre el territorio, el desarrollo local endógeno y el cooperativismo. En segundo lugar, tras una primera aproximación al posicionamiento del cooperativismo en la economía española, se define la metodología a seguir y se realiza la discusión de los resultados obtenidos a partir de su aplicación. Finalmente, se exponen las principales conclusiones y recomendaciones derivadas del análisis realizado, entre las cuales destaca el significativo peso relativo del cooperativismo en determinados sectores económicos en algunas comunidades 
autónomas donde, a priori, no se presentaban indicios sobre esa cierta importancia dentro de sus sistemas productivos, lo que requiere de análisis de carácter general y específico más rigurosos y que arrojen mayor conocimiento del sector cooperativo.

\section{TERRITORIO, DESARROLLO LOCAL ENDÓGENO Y COOPERATIVISMO}

En 1890 Alfred Marshall dio las claves teóricas del concepto de desarrollo económico local al establecer el territorio -y no la gran empresa como venían defendiendo las principales corrientes de pensamiento económico- como unidad de análisis en los procesos de desarrollo, en los que tenía lugar una aglomeración de empresas que producían mediante economías externas, constituyéndose así un enfoque estructural sistémico al no considerar elementos aislados condicionantes del desarrollo de la actividad económica en el territorio (Alburquerque, 2007).

Casi un siglo más tarde Becattini (1987), apoyado en los estudios de Marshall, observó un desarrollo que estaba teniendo lugar en determinadas regiones italianas diferente al que la corriente ortodoxa dominante explicaba: un grupo de pequeñas empresas interrelacionadas y que llevaban a cabo su actividad en el territorio estaban produciendo mediante economías externas de escala. Observó así una "industrialización difusa" en el territorio. A su vez, Piore y Sabel (1984) $)^{4}$ entre otros, ante un contexto notablemente afectado por la crisis de los años 70, defendieron que el modelo productivo basado en economías internas y generado por la gran empresa -el modelo fordista- estaba agotado. Este modelo de la gran empresa funcionaba con maquinaria muy especializada y trabajadores semicualificados que respondían de esta forma a una producción muy mecanizada y en masa. Se empezó a contemplar la necesidad de una reorganización industrial fundamentada en la producción o especialización flexible. Por otro lado, Phillipe Aydalot y el GREMI ${ }^{5}$ en los años 90 establecieron la importancia del "entorno innovador" y no del empresario individual en los procesos de desarrollo (Alburquerque, 2007) que tenían lugar en un determinado territorio, concediendo importancia a las características socioculturales presentes en éste para la difusión de la innovación.

Todas estas aportaciones presentan un núcleo común: la actividad económica está vinculada a su soporte fundamental que es el ámbito territorial en el que se vertebran los recursos endógenos en el marco específico determinado por los diferentes agentes territoriales y las relaciones entre ellos. Éste no se concibe como mero soporte físico, sino que es

\footnotetext{
${ }^{4}$ Aquí se ha trabajado con la edición española de 1990.

${ }^{5}$ Groupe de Recherche Européen sur les Milieux Innovateurs.
} 
considerado como la variable o el factor fundamental de producción y de carácter complejo al ser múltiples los factores o recursos endógenos que lo definen: la cultura, el capital social, el capital humano, los recursos naturales, los recursos financieros o las instituciones, entre otros (Garofoli, 1995; Alburquerque, 2003; Boisier, 2004; Vázquez, 2007), lo que hace que cada uno cuente con su propia función de producción a partir de sus recursos endógenos y potencialidades. Todo ello definirá la estructura productiva del territorio y su capacidad innovadora y competitiva.

Dado que el territorio es factor determinante de la función de producción, el conocimiento sobre el mismo -sus recursos y potencialidades- es condición necesaria para maximizarla. En la actualidad, las nuevas necesidades y retos sociales y medioambientales exigen que, además, se realice de manera sostenible. Por ello cobra protagonismo la comunidad local y la importancia de su participación en las estrategias de desarrollo en un doble sentido: por un lado, posee conocimiento de los recursos, potencialidades y necesidades del entorno; por otro, será la principal beneficiaria de toda política de desarrollo territorial puesta en marcha. En este sentido y siguiendo a Arocena (1995: 20), el desarrollo del territorio estará fundamentalmente condicionado por la sociedad local definida como "un sistema de acción sobre un territorio limitado, capaz de producir valores comunes y bienes localmente gestionados". Por tanto, el desarrollo local endógeno se da cuando, además de usar el potencial interno para el desarrollo, éste está liderado por los actores locales (Vázquez, 2002), y será este potencial o estos recursos endógenos los que definirán la estructura productiva o el sistema productivo local.

Las cooperativas -las organizaciones más representativas de la economía social en España- y el desarrollo local endógeno presentan elementos o características paralelas: las sociedades cooperativas nacen para dar respuesta a necesidades localizadas en un determinado territorio que, como se ha visto anteriormente, cuenta con un potencial multidimensional (económico, sociocultural, medioambiental, etc.). De esta forma, las sociedades cooperativas están vinculadas a la comunidad local de la que emergen (Calvo y González, 2011), dado que son el resultado de una dinámica social del territorio, sobre todo de la movilización de los actores sociales conscientes de los problemas que tienen lugar en su entorno (Demoustier, 2011), bien desde un interés colectivo o de un determinado grupo de personas, bien desde el interés general. 
Desde el ámbito interno de las cooperativas, uno de los rasgos o ventajas a destacar es que los procesos de toma de decisiones se basan en valores y criterios democráticos por parte de sus miembros ${ }^{6}$; desde la perspectiva externa se puede afirmar que al surgir estas actuaciones de la "dinámica endógena del territorio", las organizaciones de la economía social, y en este caso las cooperativas, a diferencia de otro tipo de empresas convencionales, no deslocalizan su actividad productiva (Demoustier, 2011:55), estando ésta muy vinculada al territorio.

A partir de sus características, las empresas cooperativas pueden comportarse como agente de transformación socioeconómica sobre todo en los territorios rurales que poseen problemas específicos -emigración, envejecimiento de la población, escasa infraestructura, baja productividad, etc.- que se traducen en niveles de vida inferiores a las áreas urbanas y en una menor competitividad económica en los mercados (Mozas y Rodríguez, 2000; Juliá y Marí, 2002; Buendía y García, 2003).

Hay que destacar que la implicación de la comunidad local en los procesos de desarrollo y la democracia en el ámbito económico (García-Gutiérrez, 1999) es una característica común tanto de la economía social en general y del cooperativismo en particular, como de los procesos de desarrollo local endógeno. La economía social en general constituye una fórmula democrática que promueve la integración y la cohesión social (Martín y Lejarriaga, 2011). Así, estas sociedades pueden constituirse como un agente de desarrollo local capaz de generar empleo, fijar la población al territorio o fomentar redes socioeconómicas, lo que se traducirá finalmente en un incremento del bienestar de los ciudadanos (Mozas y Bernal, 2006). Este impacto positivo del cooperativismo en el territorio se ha manifestado a partir de múltiples y variadas experiencias en diferentes lugares: el grupo Mondragón en País Vasco, la Cooperativa Ganadera del Valle de los Pedroches (COVAP) o las Cooperativas de Desarrollo Regional (CDR) en Quebec, son solo algunas de ellas.

Todo lo expuesto conduce a establecer que si la economía social y, en este caso particular las cooperativas, están localizadas en un determinado territorio -variable que determinará como se ha indicado en el apartado anterior, la capacidad de producción o de

\footnotetext{
${ }^{6}$ Para conocer más en profundidad los diversos modelos de gobernanza que pueden existir en las sociedades cooperativas puede consultarse el trabajo de Paredes-Frigolett, H., Nachar-Calderón, P., \& Marcuello, C. (2016). "Modeling the governance of cooperative firms". Computational and Mathematical Organization Theory, 1-45. Publicado online disponible en http://link.springer.com/article/10.1007/s10588-016-9219-z (Consultado el 10/06/2016).
} 
generación de actividad económica a partir de sus recursos endógenos-, entonces el tejido empresarial cooperativo es un factor que podrá contribuir a esa capacidad productiva y, además, lo hará de manera diferente en cada territorio en función de sus especificidades.

\section{UNA APROXIMACIÓN AL PESO DEL COOPERATIVISMO A NIVEL REGIONAL}

El territorio, su dinámica, sus recursos endógenos reales y potenciales entre los que destaca el marco empresarial e institucional público y privado, sus capacidades de reacción y crecimiento y/o sus interacciones internas y externas, determinan las características y evolución de su sistema productivo local. Este tejido empresarial depende de las condiciones y las posibilidades territoriales $\mathrm{y}$, a su vez, incide en el propio territorio, pudiendo contribuir a la dinamización de su desarrollo sostenible.

Por tanto, en este trabajo, antes de profundizar en el ámbito de las cooperativas y su contribución al sistema productivo de cada comunidad autónoma, es necesario realizar una aproximación a la situación económica general de cada una de ellas, a partir de su "valor añadido per cápita" y del "empleo" (Gráfico 1).

Gráfico 1. Tasa de empleo y valor añadido per cápita producido por la economía general en 2013.

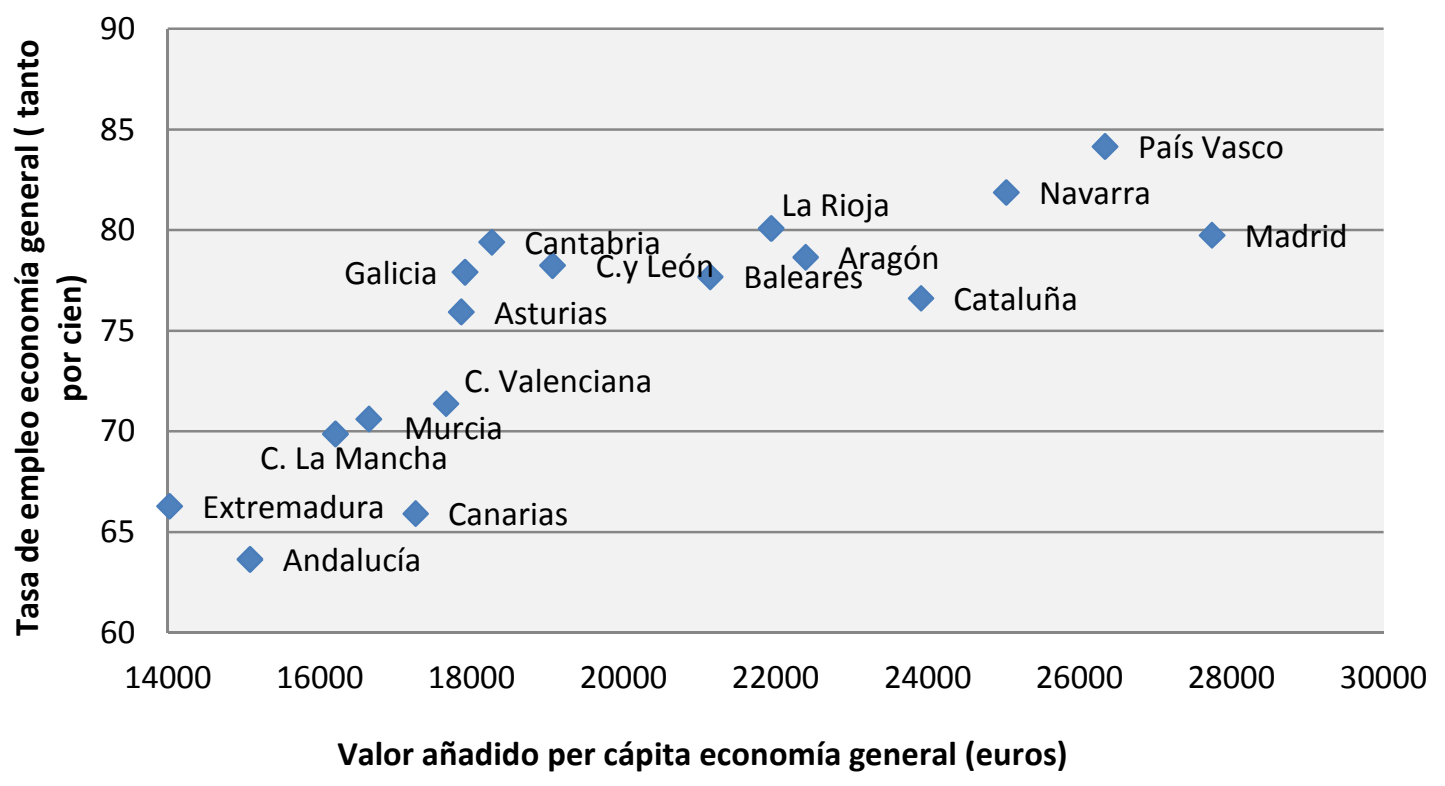

Fuente: Elaboración propia a partir de datos de la Contabilidad Nacional y de la Encuesta de Población Activa (EPA) del Instituto Nacional de Estadística (INE). 
De la interacción de las dos variables consideradas, se pone de manifiesto la existencia de una significativa heterogeneidad territorial entre las regiones, que podría agudizarse por las diferentes condiciones socioculturales, institucionales, formativas o políticas, entre otras.

Al analizar estas variables adaptadas al ámbito cooperativo ("número de empleados en cooperativas por cada 100 ocupados totales" y "valor añadido per cápita cooperativas"), resalta una fuerte diversidad regional por un lado, y una significativa diferencia respecto a los resultados obtenidos a nivel de la economía en general por otro -Madrid, Cataluña, Baleares, Extremadura y Andalucía, entre otras-. Esto pone de manifiesto, en principio, que la contribución de las cooperativas a los sistemas productivos locales no sigue las mismas pautas de la economía general en todas las comunidades autónomas y que no existe un patrón de comportamiento homogéneo del modelo cooperativo (Gráfico 2).

Gráfico 2. $\mathrm{N}^{\mathrm{o}}$ de empleados en cooperativas por cada 100 ocupados totales y valor añadido per cápita generado por las cooperativas.

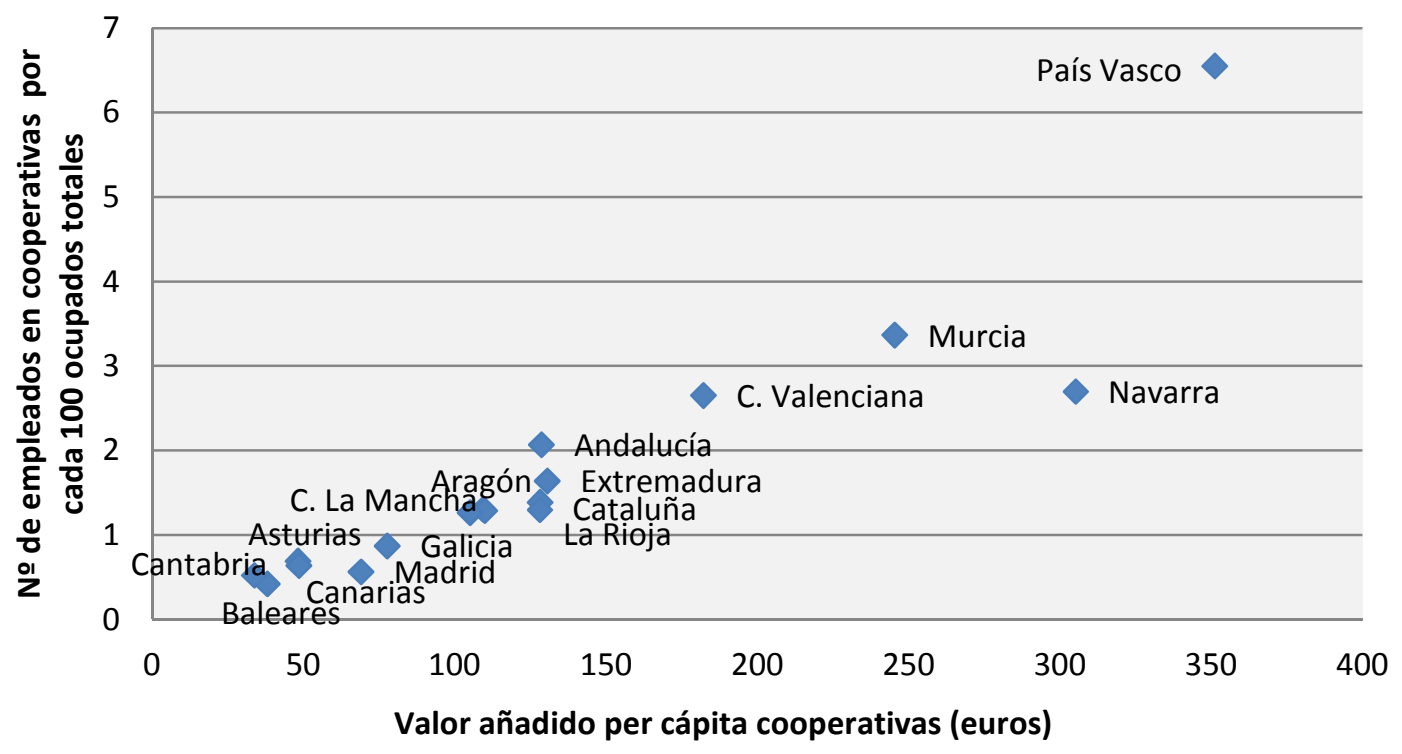

Fuente: Elaboración propia a partir de datos del Ministerio de Empleo y Seguridad Social (MEYSS).

Analizando el empleo entre las cooperativas y la economía general, hay que destacar el diferente comportamiento seguido por las comunidades autónomas y la heterogeneidad en cuanto a la incidencia del cooperativismo en los sistemas productivos locales -por ejemplo, País Vasco, con el 6,5\% de trabajadores en sociedades cooperativas, presenta un valor quince veces superior al de Baleares con el 0,42\%- . En este sentido pueden señalarse, entre otros, los 
siguientes perfiles: en primer lugar, comunidades autónomas con indicadores diferentes en cuanto a su posicionamiento a nivel económico general (Gráfico 1) pero que todas presentan valores por encima de la media en cuanto al empleo generado por las sociedades cooperativas -País Vasco, Murcia, Navarra, Comunidad Valenciana, Andalucía y Extremadura- (Gráfico 3). En segundo lugar, destacar que, aunque existen comunidades autónomas bien posicionadas en la economía general -Madrid o Baleares- (Gráfico 1), el empleo en cooperativas no es muy significativo.

Gráfico 3. Porcentaje de trabajadores en cooperativas sobre el total de ocupados en $2013^{7}$

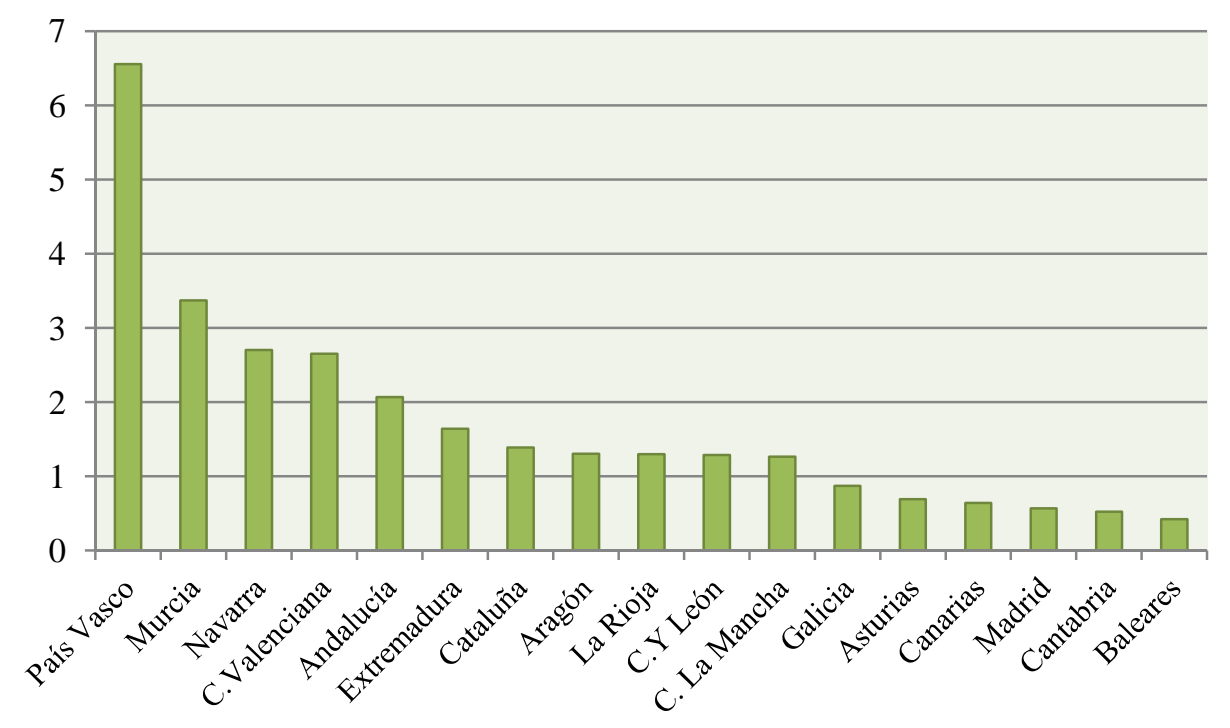

Fuente: Elaboración propia a partir de datos del Ministerio de Empleo y Seguridad Social (MEYSS) y de la Encuesta de Población Activa (EPA) del Instituto Nacional de Estadística (INE)

Por otro lado, es interesante analizar el empleo de las cooperativas por tipología de las mismas, donde destaca de manera notoria la contribución al empleo de las cooperativas de trabajo asociado en la mayoría de las comunidades autónomas y las agrarias en algunas de ellas. Ambos tipos de cooperativas -de trabajo asociado y agrarias- representan la mayor proporción de empleo generado por estas empresas -por ejemplo, en País Vasco o Andalucía, dos comunidades autónomas con sistemas productivos y dinámicas socioeconómicas diferentes, representan el $52 \%$ y el $72 \%$ respectivamente del total de empleo generado por estas sociedades-. Independientemente de la representatividad de estos dos tipos de cooperativas, se puede apreciar el comportamiento heterogéneo por territorios en cuanto al

\footnotetext{
${ }^{7}$ Para realizar un mejor análisis comparativo, el orden de las comunidades autónomas obtenido como resultado en este gráfico será el aplicado en las siguientes tablas (Tablas 1, 2 y 3 ).
} 
empleo que generan estas empresas según su clase: destacan con diferencia, respecto al resto, las comunidades autónomas de Valencia y País Vasco en cuanto a las cooperativas de consumidores y usuarios, Castilla y León en las de explotación comunitaria de la tierra, Madrid y País Vasco en enseñanza, o Aragón, Castilla La Mancha y Asturias en las de crédito (Tabla 1).

Hay que destacar que el empleo generado por las cooperativas de consumidores y usuarios en Valencia y País Vasco representa el 27,7\% y el 12,9\% respectivamente del total del empleo generado por estas empresas en cada región, poniéndose de manifiesto que en estas dos comunidades existe una cierta evidencia de la consolidación de la cultura cooperativa tanto desde la oferta como de la demanda.

Tabla 1. Porcentaje de trabajadores en sociedades cooperativas según clase

\begin{tabular}{|c|c|c|c|c|c|c|c|c|c|c|c|c|c|}
\hline CC.AA. & T.A. & C.U. & VI & AG & E.C.T & SER & $\mathbf{M}$ & TPT & SAN & ENS & CRED & OT & $\mathrm{s} / \mathrm{C}$ \\
\hline País Vasco & 50,9 & 12,9 & 0,1 & 1,5 & 0,0 & 2,1 & 0,0 & 0,0 & 0,0 & 25,6 & 1,2 & 5,7 & 0,1 \\
\hline Murcia & 39,4 & 0,0 & 0,0 & 48,6 & 1,4 & 6,6 & 0,0 & 0,3 & 0,0 & 2,5 & 0,4 & 0,8 & 0,0 \\
\hline Navarra & 34,3 & 4,8 & 0,1 & 24,0 & 0,0 & 2,2 & 0,0 & 0,5 & 0,0 & 15,8 & 18,0 & 0,3 & 0,0 \\
\hline C.Valenciana & 13,0 & 27,7 & 0,2 & 52,1 & 0,3 & 0,7 & 0,0 & 0,4 & 0,0 & 0,8 & 3,7 & 1,0 & 0,2 \\
\hline Andalucía & 40,1 & 0,7 & 0,2 & 32,5 & 1,5 & 7,0 & 0,0 & 0,1 & 0,0 & 0,1 & 16,5 & 1,1 & 0,1 \\
\hline Extremadura & 36,1 & 1,1 & 0,0 & 49,9 & 0,6 & 2,6 & 0,0 & 0,7 & 0,0 & 3,3 & 3,2 & 2,5 & 0,0 \\
\hline Cataluña & 49,5 & 7,2 & 0,1 & 16,5 & 0,0 & 5,1 & 0,0 & 0,1 & 4,0 & 9,5 & 1,4 & 6,3 & 0,1 \\
\hline Aragón & 31,0 & 2,2 & 0,1 & 30,7 & 0,8 & 7,3 & 0,0 & 1,6 & 0,0 & 2,0 & 23,1 & 1,2 & 0,0 \\
\hline La Rioja & 46,3 & 7,2 & 0,0 & 39,5 & 0,5 & 4,2 & 0,0 & 0,0 & 0,0 & 2,0 & 0,3 & 0,0 & 0,0 \\
\hline C. y León & 27,5 & 0,8 & 0,0 & 39,6 & 12,5 & 4,4 & 0,0 & 0,4 & 0,0 & 0,2 & 13,6 & 1,0 & 0,0 \\
\hline C. La Mancha & 25,2 & 1,5 & 0,5 & 44,1 & 1,2 & 1,5 & 0,0 & 0,7 & 0,0 & 1,8 & 21,1 & 1,8 & 0,5 \\
\hline Galicia & 61,6 & 0,3 & 0,0 & 20,6 & 1,5 & 2,8 & 5,4 & 1,7 & 0,0 & 3,1 & 2,2 & 0,8 & 0,0 \\
\hline Asturias & 48,1 & 0,3 & 0,2 & 15,9 & 0,4 & 3,1 & 0,0 & 3,4 & 0,0 & 5,0 & 21,1 & 2,3 & 0,0 \\
\hline Canarias & 37,1 & 4,6 & 0,1 & 39,1 & 0,4 & 6,1 & 0,1 & 3,0 & 0,0 & 1,0 & 8,5 & 0,0 & 0,0 \\
\hline Madrid & 53,8 & 2,3 & 1,8 & 1,0 & 0,0 & 8,7 & 0,0 & 0,5 & 0,0 & 30,8 & 0,1 & 1,0 & 0,0 \\
\hline Cantabria & 67,9 & 3,7 & 0,0 & 15,6 & 0,0 & 1,4 & 0,4 & 1,3 & 0,0 & 9,1 & 0,0 & 0,0 & 0,6 \\
\hline Baleares & 61,0 & 3,8 & 0,0 & 14,2 & 0,0 & 8,6 & 0,0 & 0,0 & 0,0 & 6,1 & 0,0 & 0,0 & 6,3 \\
\hline
\end{tabular}

T.A.: cooperativas de trabajo asociado, C.U.: de consumidores y usuarios, VI: de vivienda, AG.: agrarias, E.C.T.: de explotación comunitaria de la tierra, SER: de servicios, M.: de mar, TPT: de transporte, SAN.: sanitarias, ENS: de enseñanza, CRED.: de crédito, OT.: Otras, S/C: sin clasificar.

\section{Fuente: Elaboración propia a partir del Ministerio de Empleo y Seguridad Social (MEYSS)}

Estas características señaladas del modelo cooperativo en las diferentes regiones, analizado según clase de sociedad cooperativa y que evidencian una notable heterogeneidad territorial, lleva a plantear la profundización en este tema por sectores de actividad abriéndose así una nueva línea de investigación futura. 
A partir de lo anterior, para comprender mejor qué ocurre a nivel regional, es necesario un análisis más exhaustivo con el objeto de definir el peso sectorial de las sociedades cooperativas en las diferentes comunidades autónomas dada la heterogeneidad que presentan $^{8}$. Para ello, se va a partir del análisis de los coeficientes de localización sectorial tanto de las sociedades cooperativas como de la economía general medidos a través del empleo.

\subsection{Metodología}

Existen diferentes trabajos previos que estudian el comportamiento de la economía social a nivel regional español como son los de Grávalos y Pomares (2001), Clemente, Díaz y Marcuello (2009), Monzón (coord., 2010) o Pérez y Valiente (2015). Grávalos y Pomares (2001) analizan la variación del empleo y del número de empresas cooperativas a partir de una serie de variables explicativas para el periodo comprendido entre 1986 y 1995. Clemente, Díaz y Marcuello (2009), a través del análisis de convergencia propuesto por Barro y Sala i Martí, relacionan la contribución de la economía social (representada ésta por las cooperativas de trabajo asociado y las sociedades laborales) con la reducción de las desigualdades en el empleo y en el PIB desde 1999 a 2007. En el trabajo de Monzón (coord., 2010), para el periodo que abarca desde el año 2000 a 2009 y tomando como representantes de la economía social a las cooperativas de trabajo asociado y a las sociedades laborales, se estudia la evolución de estas empresas mediante un análisis descriptivo de datos disponibles en fuentes estadísticas y de la elaboración de cuestionarios a empresas del sector. Finalmente, Pérez y Valiente (2015) para los años 2007 y 2013 realizan un análisis de conglomerados o clusters para determinar la diversidad regional que existe en el nivel de autoempleo generado por la economía social. Algunas de las conclusiones a las que llegan estos trabajos son el comportamiento anticíclico de las cooperativas en las distintas regiones españolas (Grávalos y Pomares, 2001), un mayor desarrollo de la economía social en las regiones que presentan mayor dinamismo económico (Clemente, Díaz y Marcuello, 2009), la disminución de la creación de cooperativas en el periodo de la última crisis (Monzón, coord., 2010) y la inexistencia de un patrón de comportamiento homogéneo del autoempleo generado por la economía social en las diferentes comunidades autónomas españolas (Pérez y Valiente, 2015).

\footnotetext{
${ }^{8}$ El caso de País Vasco y Madrid, dos comunidades autónomas con posiciones económicas similares que cuentan con valores del empleo en cooperativas muy diferentes, es un ejemplo de esta heterogeneidad.
} 
El presente trabajo trata de reflejar el peso del cooperativismo -medido a través del empleo generado por éste, dada la importancia concedida en los últimos años a la creación de empleo estable y de calidad- en la economía regional española en un determinado momento del tiempo, en concreto para el año 2013, en plena etapa de recesión económica. Es decir, tiene por objeto mostrar la situación del empleo en sociedades cooperativas en relación a la economía de cada región ${ }^{9}$.

Para el desarrollo del análisis planteado, tras una aproximación a nivel regional a partir del valor añadido per cápita y del empleo, se ha elaborado un indicador o ratio $\left(W_{i j}\right)$ que pone en relación el coeficiente de localización sectorial del cooperativismo $\left(C L \operatorname{coop}_{i j}\right)$ con el coeficiente de localización sectorial de la economía general $\left(C L_{i j}\right)$, medidos a través del empleo. Estos coeficientes tienen por objeto determinar la distribución regional o el grado de concentración de un sector productivo en un territorio con respecto a la distribución o concentración de este sector en el territorio tomado como referencia. En este caso se trata de establecer el grado de concentración en los diferentes sectores productivos del empleo generado por las cooperativas en las diferentes comunidades autónomas en relación al generado a nivel nacional.

Si el coeficiente de localización sectorial descrito toma valores superiores a 1 para un determinado sector en una región dada, entonces sí puede decirse que existe una especialización o localización relativa del sector objeto de estudio en el territorio de referencia. De esta forma, el coeficiente de localización sectorial para el cooperativismo se define mediante la siguiente expresión:

$$
C L \operatorname{coop} p_{i j}=\frac{\frac{E \operatorname{coop}}{E \operatorname{coop}} p_{j}}{\frac{E \operatorname{coop} p_{i}}{E \operatorname{coop} p_{t}}}
$$

siendo:

$\operatorname{CLCoop}_{i j}=$ coeficiente de localización del cooperativismo del sector $i$ en la región $j$, $E \operatorname{coop}_{i j}=$ empleo generado en las cooperativas por el sector $i$ en la región $j, E \operatorname{coop}_{j}=$ empleo total generado en las cooperativas en la región j, $E \operatorname{coop}_{i}=$ empleo total generado en las

\footnotetext{
${ }^{9}$ No se han considerado las ciudades autónomas de Ceuta y Melilla por su situación particular.
} 
cooperativas en el sector $i$ en el total de regiones o en la economía española, $E \operatorname{coop}_{t}=$ empleo total generado en las cooperativas en la economía española.

En cuanto al coeficiente de especialización o localización de la economía general, éste queda definido a través de las siguientes relaciones:

$$
C L_{i j}=\frac{\frac{E_{i j}}{E_{j}}}{\frac{E_{i}}{E_{t}}}
$$

siendo:

$C L_{i j}=$ coeficiente de localización de la economía del sector $i$ en la región $j, \quad E_{i j}=$ empleo generado por el sector $i$ en la región $j, E_{j}=$ empleo total generado en la región $\mathrm{j}, E_{i}=$ empleo total generado en el sector $i$ en el total de regiones o a nivel nacional, $E_{t}=$ empleo total generado en la economía española. De esta forma se conoce el peso de cada sector en la estructura productiva de cada comunidad autónoma, es decir, la importancia de los diferentes sectores dentro de una comunidad autónoma respecto al mismo sector a nivel nacional desde la perspectiva del empleo.

A partir de estos ratios, en el presente trabajo, se propone el indicador del peso del cooperativismo $W_{i j}$ derivado de la relación entre los coeficientes de localización sectorial planteados arriba:

$$
W_{i j}=\frac{C L \operatorname{coop}_{i j}}{C L_{i j}}
$$

representando $W_{i j}$ el indicador del peso del cooperativismo -medido a través del empleo generado- en el sector $i$ de la región $j$. Si $W_{i j}>1$, entonces el peso del cooperativismo es muy significativo en el sector y en la región objeto de análisis, es decir, el empleo en sociedades cooperativas está más localizado o concentrado en un determinado sector del territorio, que el empleo de este mismo sector en la economía general. Por el contrario, si $W_{i j}<1$, significa que el cooperativismo no presenta un peso importante en el sector productivo de la región en relación a la especialización general de este sector en el territorio.

Los datos empleados para la elaboración del indicador propuesto en este trabajo se han obtenido del Ministerio de Empleo y Seguridad Social (MEYSS) y del Instituto Nacional de 
Estadística (INE). Del MEYSS se han extraído el número de trabajadores totales, incluidos los trabajadores autónomos, en sociedades cooperativas por sectores productivos. Del INE se ha obtenido el número de ocupados por sectores económicos y por comunidades autónomas, a partir de la Encuesta de Población Activa (EPA). Todos los datos están referidos al año 2013.

\subsection{Resultados y discusión}

A partir del análisis del coeficiente de localización de la economía general $\left(C L_{i j}\right)$ se obtiene una primera aproximación de la especialización sectorial en las diferentes comunidades autónomas -medida a través del empleo-, poniéndose de manifiesto las comunidades que más destacan en cada uno de los sectores productivos (Tabla 2).

Tabla 2. Coeficiente de especialización de la economía general $\left(C L_{i j}=\frac{E_{i j}}{E_{j}} / \frac{E_{i}}{E_{t}}\right)$

\begin{tabular}{|c|r|r|r|r|}
\hline Región & \multicolumn{1}{|c|}{$\begin{array}{c}\text { Sector } \\
\text { primario }\end{array}$} & Industria & Construcción & Servicios \\
\hline País Vasco & 0,38 & 1,49 & 1,08 & 0,94 \\
\hline Murcia & 3,08 & 0,96 & 0,91 & 0,90 \\
\hline Navarra & 1,36 & 1,90 & 0,92 & 0,82 \\
\hline C. Valenciana & 0,78 & 1,22 & 0,99 & 0,97 \\
\hline Andalucía & 1,77 & 0,62 & 0,87 & 1,03 \\
\hline Extremadura & 2,37 & 0,78 & 1,14 & 0,95 \\
\hline Cataluña & 0,41 & 1,34 & 1,03 & 0,97 \\
\hline Aragón & 1,38 & 1,25 & 1,00 & 0,93 \\
\hline La Rioja & 1,63 & 1,93 & 1,04 & 0,79 \\
\hline C. y León & 1,67 & 1,12 & 1,20 & 0,92 \\
\hline C. La Mancha & 1,69 & 1,15 & 1,24 & 0,91 \\
\hline Galicia & 1,72 & 1,11 & 1,15 & 0,93 \\
\hline Asturias & 1,10 & 1,07 & 1,06 & 0,98 \\
\hline Canarias & 0,75 & 0,39 & 0,94 & 1,13 \\
\hline Madrid & 0,10 & 0,67 & 0,84 & 1,12 \\
\hline Cantabria & 0,89 & 1,21 & 1,21 & 0,95 \\
\hline Baleares & 0,24 & 0,41 & 1,29 & 1,13 \\
\hline
\end{tabular}

Fuente: Elaboración propia

Entre los resultados obtenidos para este coeficiente de localización y en lo que respecta al sector primario, son las comunidades de Murcia y Extremadura las que destacan con valores superiores al resto, lo que significa que, dentro de estas regiones, la proporción de empleo dirigido al sector primario es mayor que la proporción dirigida a este sector a nivel español. Lo mismo ocurre para las comunidades autónomas de País Vasco y Navarra en el sector industrial; para Baleares o Castilla La Mancha en la construcción; y para Canarias, Baleares y Madrid en el sector servicios (Tabla 2). 
Una vez planteada la especialización productiva de cada comunidad autónoma medida a través del empleo en general -es decir, sin distinguir el tipo de empresa o entidad que lo genera-, se ha analizado el peso del cooperativismo definido por $W_{i j}$ para profundizar en la determinación de la concentración del cooperativismo a nivel territorial en España.

Tabla 3. Peso del cooperativismo $\left(W_{i j}=C L \operatorname{coop} p_{i j} / C L_{i j}\right)$

\begin{tabular}{|c|c|c|c|c|}
\hline Región & $\begin{array}{c}\text { Sector } \\
\text { primario }\end{array}$ & Industria & Construcción & Servicios \\
\hline País Vasco & 0,08 & 1,18 & 0,93 & 0,98 \\
\hline Murcia & 0,78 & 0,34 & 1,62 & 1,06 \\
\hline Navarra & 0,38 & 0,89 & 3,28 & 0,93 \\
\hline C.Valenciana & 0,28 & 0,34 & 0,53 & 1,04 \\
\hline Andalucía & 0,81 & 1,29 & 0,98 & 0,96 \\
\hline Extremadura & 0,47 & 1,41 & 0,95 & 0,99 \\
\hline Cataluña & 0,54 & 0,75 & 1,18 & 1,16 \\
\hline Aragón & 0,43 & 1,12 & 1,24 & 1,11 \\
\hline La Rioja & 0,07 & 0,91 & 1,61 & 0,78 \\
\hline C. y León & 1,04 & 1,29 & 0,86 & 0,80 \\
\hline C. La Mancha & 0,78 & 1,39 & 0,98 & 0,89 \\
\hline Galicia & 0,50 & 1,32 & 1,57 & 1,29 \\
\hline Asturias & 0,60 & 0,40 & 0,80 & 1,12 \\
\hline Canarias & 0,51 & 1,56 & 0,35 & 1,28 \\
\hline Madrid & 0,40 & 0,45 & 0,48 & 1,27 \\
\hline Cantabria & 0,24 & 0,62 & 1,09 & 1,29 \\
\hline Baleares & 0,29 & 0,46 & 0,42 & \\
\hline & & & & \\
\hline & 0 & & \\
\hline
\end{tabular}

Fuente: Elaboración propia

La aplicación de este indicador más específico arroja unos resultados que profundizan los obtenidos en el primer análisis anterior y que en algunos escenarios ofrecen conclusiones que, a priori, no pueden derivarse solo del primer análisis de carácter más general.

En primer lugar, el peso del cooperativismo en el sector primario no es significativo (salvo en la comunidad de Castilla y León). Esto significa que el coeficiente de localización de la economía general es superior al coeficiente de localización del cooperativismo, lo que quiere decir que la proporción relativa de trabajadores en empresas convencionales del sector primario con respecto a la proporción a nivel nacional, es mayor que la de los trabajadores en cooperativas. Aunque son numerosas las cooperativas agrarias en nuestro país -en 2013 unas 3.379 representando cerca del 29\% del total de estas empresas según el Ministerio de Empleo y Seguridad Social (MEYSS)- éste no es un sector al que especialmente se dirija el empleo generado por estas sociedades en relación al resto de las organizaciones, es decir, no destaca 
en relación al sector empresarial tradicional o convencional (Tabla 3), a pesar de que tradicionalmente la fuerte presencia de las cooperativas en el sector agrario español ha sido significativa.

En segundo lugar, el peso relativo del cooperativismo en el sector industrial sí está más presente que en el sector primario. Éste está marcado por un peso relativo del cooperativismo importante en las comunidades de País Vasco, Andalucía, Extremadura, Aragón, Castilla y León, Castilla la Mancha, Galicia y Canarias. Se trata de un resultado interesante dado que las comunidades autónomas de Andalucía o Extremadura por ejemplo, cuentan con muy bajo nivel de especialización productiva en el sector industrial $(0,62$ y 0,78 respectivamente) (Tabla 2), pero, sin embargo, existen una serie de factores que determinan una especial localización del empleo de las cooperativas en este sector superior al de empresas convencionales de la industria. El indicador del peso del cooperativismo de estas dos comunidades tiene valores superiores incluso a los de País Vasco $(1,49)$, comunidad autónoma con un elevado índice de especialización productiva en la industria (Tabla 2).

Tabla 4. Estadísticos principales del peso del cooperativismo $\left(W_{i j}\right)$

\begin{tabular}{|c|c|c|c|c|}
\hline & $\begin{array}{c}\text { Sector } \\
\text { primario }\end{array}$ & Industria & Construcción & Servicios \\
\hline Media & 0,48 & 0,92 & 1,11 & 1,05 \\
\hline Mediana & 0,47 & 0,91 & 0,98 & 1,04 \\
\hline Mínimo & 0,07 & 0,34 & 0,35 & 0,78 \\
\hline Máximo & 1,04 & 1,56 & 3,28 & 1,29 \\
\hline Desv. típica & 0,26 & 0,42 & 0,68 & 0,16 \\
\hline Coef. variación & 0,54 & 0,46 & 0,61 & 0,15 \\
\hline Asimetría & 0,34 & $-0,10$ & 1,87 & 0,03 \\
\hline Exc. de curtosis & $-0,39$ & $-1,49$ & 4,02 & $-0,98$ \\
\hline
\end{tabular}

Fuente: Elaboración propia

En tercer lugar, en el sector de la construcción, y especialmente en la comunidad de Navarra, el peso del cooperativismo también destaca siendo su coeficiente de localización de más del triple que el de la economía general (Tabla 3). Dado que los resultados obtenidos son para el año 2013, en un contexto de crisis económica en el que España experimenta una caída del Producto Interior Bruto del 1,1\% según datos del Instituto Nacional de Estadística (INE), y en el que el sector de la construcción se ha visto especialmente afectado, aunque se requiere de un estudio en profundidad, esto podría ser un indicio de lo que ya estudios anteriores en diferentes contextos como los de Tomas Carpi (1997), Grávalos y Pomares (2001) o Díaz y 
Marcuello (2010) han demostrado: la mayor resistencia del empleo en organizaciones de la economía social frente a organizaciones tradicionales de la economía en general, ante variaciones del ciclo económico; o en el caso de la creación de cooperativas, los trabajos de Rusell y Hanneman (1992) o de Pérotin (2006) señalan que, aunque las cooperativas y el resto del tejido empresarial presentan un comportamiento semejante en lo que respecta a la salida del mercado de estas organizaciones, no ocurre lo mismo con la creación de nuevas sociedades cooperativas que sí presentan un comportamiento contracíclico.

No obstante, hay que señalar también que es en el sector de la construcción donde existe mayor heterogeneidad del peso del cooperativismo $\left(W_{i j}\right)$, siendo su coeficiente de variación del 0,61 (Tabla 4). 
Gráfico 4. Peso del cooperativismo $\left(W_{i j}=C L c o o p_{i j} / C L_{i j}\right)$ por comunidades autónomas españolas, 2013.
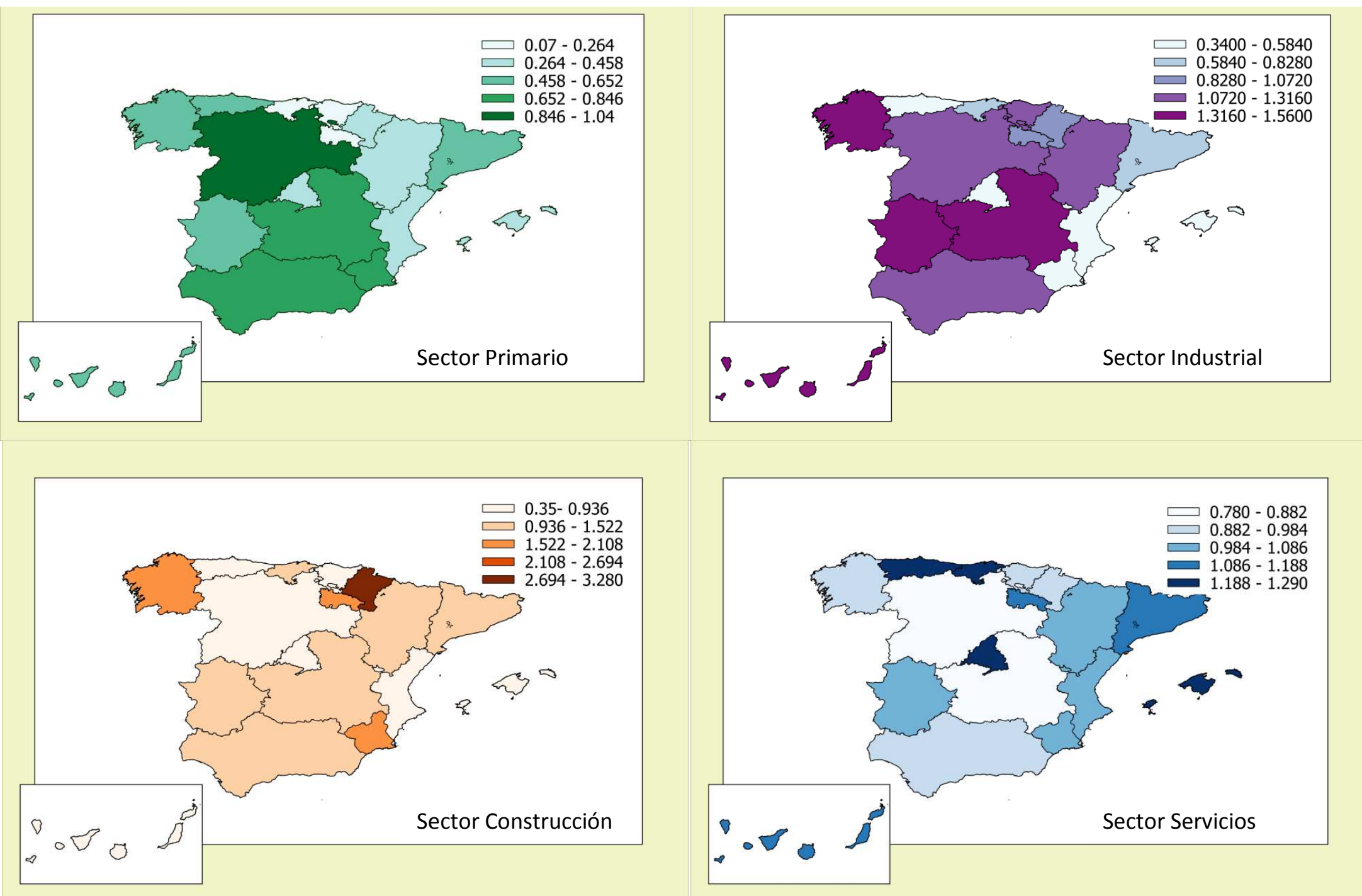

Fuente: Elaboración propia

REVESCO Nº 123 - Primer Cuatrimestre 2017 - ISSN: 1885-8031 - www.ucm.es/info/revesco 
Finalmente, es en el sector servicios donde el indicador del peso del cooperativismo presenta valores superiores a 1 en más de la mitad de las comunidades autónomas. Cuando se ha analizado a nivel general el número de trabajadores en cooperativas en proporción al número de trabajadores totales en la economía (Gráfico 3), se ha obtenido que las comunidades de Asturias, Canarias, Madrid, Cantabria y Baleares están a la cola presentando los valores más bajos en número de trabajadores relativos en cooperativas. Sin embargo, en el sector servicios de estas regiones, el indicador del peso del cooperativismo es mayor que 1, demostrando así un coeficiente de localización para el empleo en las cooperativas superior al de la economía general.

Este sector, el de servicios, es el que presenta la menor dispersión del peso relativo del empleo en las sociedades cooperativas en relación al de la economía general ya que su coeficiente de variación tiene un valor de 0,15 inferior al del resto de los sectores, y con una media y mediana casi similares (1,05 y 1,04 respectivamente), lo que significa que la muestra es más homogénea que en el resto de los sectores (Tabla 4). 
Gráfico 5. Ilustración gráfica del peso del cooperativismo $\left(W_{i j}\right)$

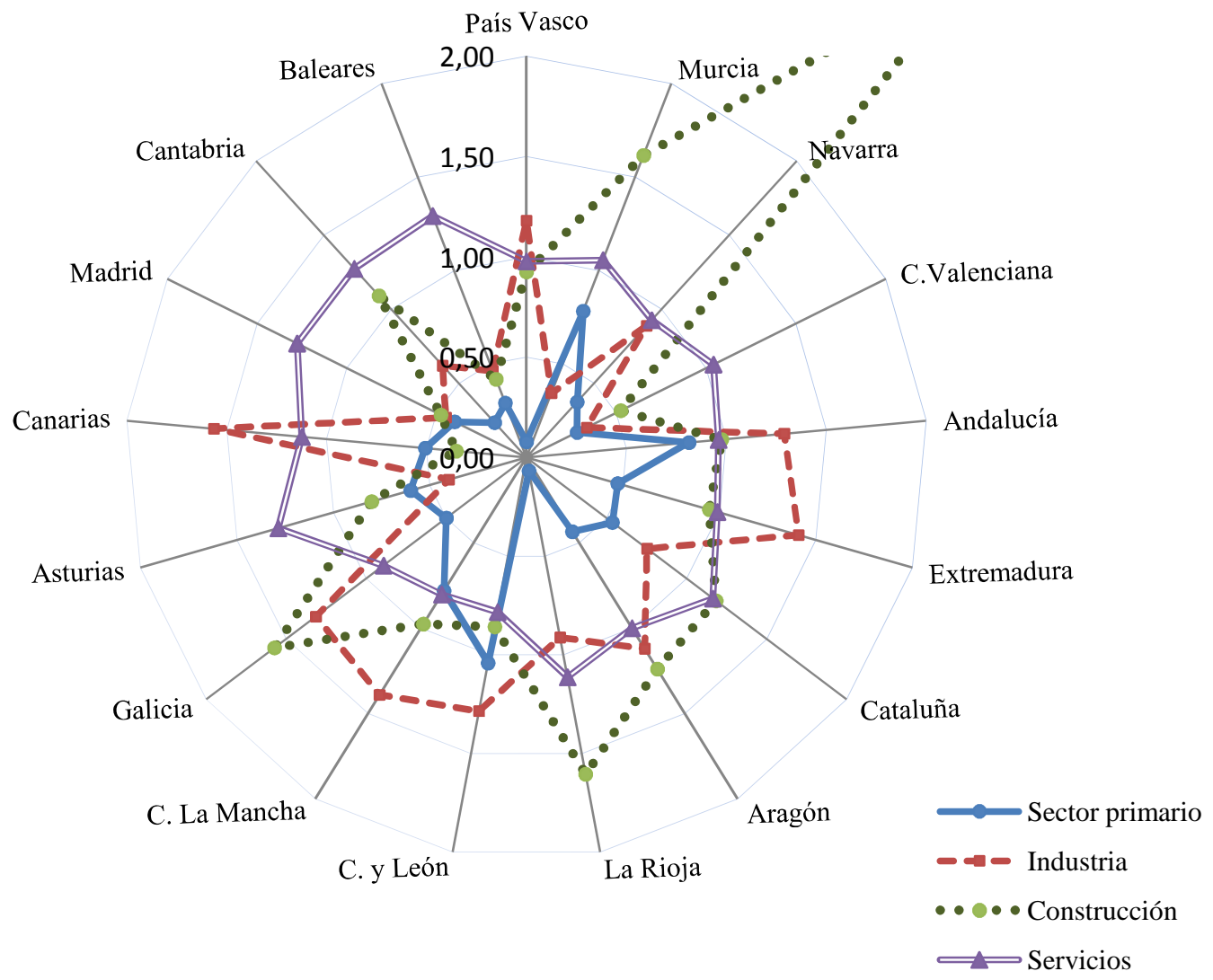

Fuente: Elaboración propia

A partir del análisis anterior, hay que destacar la heterogeneidad sectorial y territorial en el peso del cooperativismo $\left(W_{i j}\right)$, en las diferentes comunidades autónomas (Gráfico 4). Aunque en el sector servicios presenta cierta homogeneidad, en términos generales no existe un patrón de comportamiento definido en el territorio español (Gráfico 5). Por tanto, hay que resaltar una heterogeneidad territorial que se manifiesta tanto en los sistemas productivos locales como en los modelos cooperativos.

\section{CONCLUSIONES FINALES}

Es cada vez mayor la importancia que están teniendo las organizaciones de la economía social en general y las cooperativas en particular, tanto desde el ámbito científico como institucional, sobre todo durante la última crisis que puede ser considerada como una oportunidad de cambio de los modelos productivos territoriales en busca de la sostenibilidad socioeconómica, medioambiental y territorial. 
Los valores, principios y las especificidades de las cooperativas, -las empresas más numerosas y representativas dentro de la economía social en España-, las configuran como agentes socioeconómicos que pueden tener una considerable contribución al tejido empresarial, al sistema productivo territorial y al desarrollo endógeno sostenible, por su implicación con el resto de agentes territoriales. Pero la participación de las cooperativas en el entorno socioeconómico donde desarrolla su actividad, no es homogénea en las diferentes comunidades autónomas españolas, sino que puede depender de la realidad territorial y de su potencialidad, vertebrándose de manera diversa según lo anterior y, por tanto, teniendo un diferente impacto en el territorio.

En este sentido, es necesario conocer por un lado, el modelo cooperativo en las diferentes regiones; y, por otro, las características y especificidades territoriales de cada una de ellas, para poder diseñar y desarrollar líneas estratégicas integradas, que permitan la generación de sinergias dentro de cada territorio y en sus relaciones con otros espacios, con mayor garantía de resultados.

Para profundizar en el conocimiento de estos modelos cooperativos a partir de la realidad socioeconómica de cada comunidad autónoma, se ha elaborado el índice del peso del cooperativismo $\left(W_{i j}\right)$, a fin de identificar a qué sectores se dirige en cada región y su comportamiento respecto al resto del tejido empresarial dentro del sistema productivo territorial; es decir, se ha determinado el grado de concentración en los diferentes sectores productivos del empleo generado por las cooperativas en las comunidades autónomas, en relación al generado a nivel nacional.

A nivel general y previamente a los resultados obtenidos por el índice del peso del cooperativismo, hay que señalar que la generación de empleo y de valor añadido per cápita por parte de estas sociedades presenta un comportamiento heterogéneo entre las comunidades autónomas españolas (Gráfico 2). Además, si se atiende al valor añadido y al empleo generado por la economía general por un lado (Gráfico 1), y por las cooperativas por otro (Gráfico 2), no puede señalarse que exista de manera general una relación ni directa ni inversa entre estos dos ámbitos: es decir, valores altos en la economía general no implican necesariamente valores igualmente elevados en las cooperativas, ni que valores altos en la primera incidan en valores bajos en las segundas, y viceversa, sino que dependerá de cada comunidad autónoma. En este sentido, caben señalar las comunidades de País Vasco y Madrid que aunque presentan ambas elevados posicionamientos económicos en la economía 
general -en este caso empleo y valor añadido per cápita- (Gráfico 1), los resultados relativos al cooperativismo son diferentes: mientras que para País Vasco éstos son altos también, para el caso de Madrid las variables indican un nivel de cooperativismo inferior al de otras comunidades como Andalucía o Extremadura con indicadores económicos significativamente más bajos (Gráfico 2).

Por otro lado, la heterogeneidad manifestada anteriormente se ha visto reforzada al realizar el análisis del empleo generado por las sociedades cooperativas según su clase. Por ejemplo, País Vasco y Comunidad Valenciana, dos regiones con dinámicas socioeconómicas diferentes, presentan una proporción de trabajadores en las cooperativas de consumidores y usuarios muy superiores al resto de comunidades (Tabla 1).

En lo que respecta a los resultados obtenidos a partir del índice del peso del cooperativismo $\left(W_{i j}\right)$, éstos han manifestado que en regiones en las cuales, a priori y a nivel general, existía una escasa importancia en el empleo generado por las empresas cooperativas (Gráfico 3), cuando se han analizado con mayor profundidad los diferentes sectores y territorios en relación con la economía general mediante la aplicación de dicho índice, esta consideración cambia: existen comunidades autónomas como Cantabria y Baleares que, aunque en cuanto al porcentaje de trabajadores en cooperativas sobre el total de ocupados se sitúan a la cola de España, el indicador del peso del cooperativismo propuesto en este trabajo pone de manifiesto un coeficiente más elevado que el de la economía general, concretamente en el sector servicios, mostrando así un mayor peso de las cooperativas de lo que a priori pudiera derivarse.

Según este indicador aplicado, por sectores, han destacado las comunidades autónomas de Castilla y León $(1,04)$ en las actividades primarias; Canarias $(1,56)$, Extremadura $(1,41)$ y Castilla La Mancha $(1,39)$ en la industria; Navarra $(3,28)$, Murcia $(1,62)$ y La Rioja $(1,61)$ en la construcción; y Baleares $(1,29)$, Asturias $(1,29)$ y Madrid $(1,28)$ en el sector servicios, entre otras (Gráfico 4).

A partir de lo expuesto, se puede sostener que existe heterogeneidad en cuanto a los diferentes modelos cooperativos que puedan encontrarse en las comunidades autónomas españolas. Esto manifiesta que podrían existir determinadas características o factores -no estrictamente relacionados con indicadores económicos generales como el valor añadido o el 
nivel de empleo- que influyen de manera positiva en la localización de estas organizaciones en los diferentes sectores en relación a la economía general.

La diferencia en los sistemas productivos locales y en el cooperativismo entre las diferentes comunidades autónomas podrían incidir y, a su vez, ser consecuencia de la diversidad territorial; es decir, cada territorio es definido a partir de unos recursos, características o especificidades propias que determinan su sistema productivo local $\mathrm{y}$, al mismo tiempo, este sistema productivo tiene un impacto concreto en el territorio, aspectos que deben considerarse al diseñar y aplicar políticas y estrategias encaminadas a fomentar, consolidar y mejorar la contribución de las sociedades cooperativas al desarrollo territorial sostenible.

El presente trabajo ha sentado una base para analizar en profundidad por qué el peso del cooperativismo destaca más en unas regiones que en otras, abriendo así una futura línea de investigación para determinar la relación que existe entre el peso del cooperativismo y otras variables -no estrictamente económicas- que definan el territorio desde una perspectiva multidimensional, es decir, social, económica, institucional, cultural y/o ambiental, respondiendo sobre todo a la cuestión de qué factores o características promueven la existencia de determinados modelos cooperativos. Por otro lado, se establece también como futura línea de investigación analizar el peso del cooperativismo por clases de cooperativas y sector económico en el que desempeñan su actividad, teniendo en cuenta la limitación en cuanto a los datos existentes.

En definitiva, se ha puesto de manifiesto a lo largo del presente trabajo, el comportamiento heterogéneo que presentan los modelos cooperativos en las distintas comunidades autónomas españolas, sin que pueda establecerse un patrón general de comportamiento a nivel español y siendo necesario por ello análisis exhaustivos a nivel territorial de cada una de ellas.

\section{BIBLIOGRAFÍA}

ALBURQUERQUE, F. (2003) Teoría y práctica del enfoque del desarrollo local. Instituto de Economía y Geografía. Consejo Superior de Investigaciones Científicas, Madrid. http://www.ciieberazategui.com.ar/junio2013/noviembre2013/Falburquerque-

Coquimbo_Masregion.pdf (Consultado el 1/02/2015). 
ALBURQUERQUE, F. (2007) Desarrollo económico y territorio: Enfoques teóricos relevantes y reflexiones derivadas de la práctica. En M. García (Ed.), Perspectivas teóricas en Desarrollo Local, pp. 59-74. La Coruña: Netbiblo.

AROCENA, J. (1995) El desarrollo local: un desafío contemporáneo. Caracas: Nueva Sociedad.

BECATTINI, G. (1987) Mercato e forze locali: il distretto industriale. Bolonia, Il Mulino.

BOISIER, S. (2004) Desarrollo territorial y descentralización: el desarrollo en el lugar y en las manos de la gente. Eure (Santiago), Vol. 30, № 90, pp. 27-40.

BUENDIA, I. y GARCIA J. V. (2003) Cooperativismo y desarrollo local. Madrid: Editorial Centro de Estudios Ramón Areces.

CALVO, R. Y GONZÁLEZ, J. (2011) La creación de empresas de economía social en el modelo de desarrollo local: reflexiones sobre la divergencia actual del caso valenciano. REVESCO. Revista de Estudios Cooperativos, $\mathrm{N}^{\mathrm{o}}$ 104, pp. 7-37. DOI: 10.5209/rev_REVE.2011.v104.1.

CLEMENTE, J., DÍAZ, M. y MARCUELLO, C. (2009) Sociedades cooperativas y sociedades laborales en España: estudio de su contribución a la creación de empleo y al crecimiento económico. REVESCO. Revista de Estudios cooperativos, № 98, pp. 35-69.

CORAGGIO, J.L. (2011) Economía Social y Solidaria: el trabajo antes que el capital. Quito: Abya-Yala.

DEMOUSTIER, D. (2011) Les concepts philosophiques et sociaux de l'ESS dans ses rapports à l'activité économique et aux territoires. En A. Ndiaye (dir.), Économie sociale et solidaire: animation et dynamiques des territoires, pp. 47-60. Paris: L'Harmattan.

DÍAZ, M. y MARCUELLO, C. (2010) Impacto económico de las cooperativas. La generación de empleo en las sociedades cooperativas y su relación con el PIB. CIRIECEspaña, Revista de Economía Pública, Social y Cooperativa, № 67, pp. 23-44.

GARCÍA-GUTIÉRREZ, C. (1999). Cooperativismo y desarrollo local. REVESCO, Revista de Estudios Cooperativos, $\mathrm{N}^{\mathrm{0}}$ 68, pp. 33-46.

GAROFOLI, G. (1995) Desarrollo económico, organización de la producción y territorio. En A. Vázquez y G. Garófoli, Desarrollo económico local en Europa, p. 113-123. Madrid: Colegio de Economistas.

GRÁVALOS, M.A. y POMARES, I. (2001) Cooperativas, desempleo y efecto refugio. REVESCO. Revista de Estudios Cooperativos, № 74, pp. 69-84. 
GURIDI, L. y PÉREZ DE MENDIGUREN, J.C. (2014) La dimensión económica del Desarrollo Humano Local: La economía social y solidaria. Hegoa: Bilbao. http://www.dhl.hegoa.ehu.es/ficheros/0000/0781/ESSDHL.pdf.

(Consultado el 29/01/2016).

JORDÁN, J., ANTUÑANO, I. y FUENTES, V (2013) Desarrollo endógeno y política anticrisis. Ciriec-España Revista de Economía Pública, Social y Cooperativa, No 78, pp. 245-263.

JULIÁ, J., y MARÍ, S. (2002) Agricultura y desarrollo rural. CIRIEC-España, Revista de Economía Pública, Social y Cooperativa, № 48, pp. 25-52.

MARTÍN, S. Y LEJARRIAGA, G. (2011) Las empresas de participación de trabajo asociado ante los desafíos del futuro en un contexto de crisis económica: propuestas de actuación. CIRIEC-España, Revista de Economía Pública, Social y Cooperativa, № 56, pp. 9-24.

MELIÁN, A. y CAMPOS, V. (2010) Emprendedurismo y economía social como mecanismos de inserción sociolaboral en tiempos de crisis. REVESCO. Revista de Estudios Cooperativos, $\mathrm{N}^{\circ} 100, \mathrm{pp} .43-67$.

MONZÓN, J.L. (Coord.) (2010) Las empresas de trabajo asociado en España. Evolución reciente y perspectivas. Valencia: CIRIEC-España editorial.

MOZAS, A. y BERNAL, E. (2006) Desarrollo territorial y economía social. CIRIEC- España, Revista de Economía Pública, Social y Cooperativa, No 55, pp. 125-140.

MOZAS, A. y RODRIGUEZ, J. C. (2000) La economía social: agente del cambio estructural en el ámbito rural. Revista de Desarrollo Rural y Cooperativismo Agrario, No 4, pp. 7- 18. PAREDES-FRIGOLETT, H., NACHAR-CALDERÓN, P., \& MARCUELLO, C. (2016) Modeling the governance of cooperative firms. Computational and Mathematical Organization Theory, 1-45. Publicado online disponible en: http://link.springer.com/article/10.1007/s10588-016-9219-z (Consultado el 10/06/2016).

PÉREZ, M.C. y VALIENTE, L. (2015) Impacto territorial del autoempleo en la economía social en España. CIRIEC-España, Revista de Economía Pública, Social y Cooperativa, $\mathrm{N}^{\circ} 83$, pp. 83-114.

PÉROTIN, V. (2006) Entry, exit and the business cycle. Are cooperatives different? Journal of Comparative Economics, $N^{\circ} 34$, pp. 295-316.

PIORE, M. y SABEL, C. (1990) La segunda ruptura industrial. Madrid: Alianza Editorial. TOMÁS CARPI, J.A. (1997) La economía social en un mundo en transformación. CIRIECEspaña, Revista de Economía Pública, Social y Cooperativa, № 25, pp. 83-115. 
RUSSELL, R. \& HANNEMAN, R. (1992) Cooperatives and the business cycle: The Israeli case. Journal of Comparative Economics, Vol. 16, № 4, pp. 701-715.

VÁZQUEZ, A. (2002) Endogenous Development: Networking, Innovation, Institutions and Cities. New York: Routledge studies in developmental economics.

VÁZQUEZ, A. (2007) Desarrollo endógeno. Teorías y políticas de desarrollo territorial. Investigaciones Regionales, № 11, pp. 183-210. 